\title{
Alpha-1-Antitrypsin-Mangel
}

\section{Mit umfassendem Therapiesystem früh erkennen und versorgen}

— Die Erbkrankheit Alpha-1-Antitrypsin-Mangel (AATM) ist gekennzeichnet durch einen unzureichenden Schutz alveolärer Strukturen durch das Protein Alpha-1-Antitrypsin und dadurch fortwährende Schädigungen der Lunge durch entzündliche Prozesse. „Die Symptome des AATM ähneln denen der klassischen COPD“, erklärte Dr. Heinz Steveling, Leitender Oberarzt der Abteilung Pneumologie am Universitätsklinikum Essen. „Dadurch wird die Krankheitsursache häufig erst spät oder gar nicht erkannt."

\section{Unterstützung bei Diagnose und Therapie}

Für die Aufklärung von Ärzten und Patienten zum AATM setzt sich die Initiative PROAlpha ein, eine Partnerschaft von Al-
pha-1-Zentren, interessierten Ärzten, Selbsthilfegruppen und dem Unternehmen Grifols. Die Initiative stützt sich auf nationale und internationale Leitlinien, wonach jeder COPD-Patient einmal im Leben auf einen AATM zu testen ist.

Über die Webseite von PROAlpha (www.initiative-pro-alpha.de) kann der Arzt den Blutschnelltest AlphaKit ${ }^{\circ}$ QuickScreen anfordern, mit dem das Vorliegen des Z-Allels - die häufigste Ursache für einen AATM - nach 15 Minuten mit hoher Wahrscheinlichkeit ausgeschlossen werden kann. Ist das Testergebnis positiv, kann eine Bestimmung des Geno- und Phänotyps am Alpha1-Labor in Marburg mittels des ebenfalls kostenfreien AlphaKit ${ }^{\circ}$ erfolgen.

Die Substitutionstherapie mit Prolastin $^{\infty}$ (humanes Alpha-1-Antitrypsin) steht für Alpha-1-Patienten mit bestimmten Phänotypen, mittelgradiger Störung der Lungenfunktion $\left(\mathrm{FEV}_{1} 35\right.$ $60 \%$ ) und nach Überprüfung des klinischen Zustands (Grad der Beeinträchtigung) zur Verfügung. Dadurch kann der Verlust an Lungengewebe verlangsamt werden.

\section{Hilfestellung im Patientenalltag}

Für die besonderen Bedürfnisse von AATM-Patienten und ihren Angehörigen bietet das Serviceprogramm AlphaCare (www.alpha-care.de) neben fundierten Informationen auch Unterstützungsangebote für das Alltagsleben.

Gudrun Girrbach

- Pressegespräch „Alpha-1-Antitrypsin-Mangel: Optimierte Versorgung von Alpha-1-Patienten durch umfassendes Therapiesystem"; Düsseldorf, Februar 2016 (Veranstalter: Grifols)

\section{Individualisierte Therapie mit doppelter Steroiddosis}

\section{Allergisches Asthma - eine Zivilisationskrankheit}

_ Früher war der Präventionsgedanke davon geprägt, Allergene zu meiden, so dass z. B. Kinder keinen Hamster halten durften. Dies ist laut Prof. Matthias Kopp, Lübeck, heute überholt, denn Studien hätten gezeigt, dass der Kontakt zu Hunden und Nagern in den ersten Lebensjahren vor Sensibilisierung schütze. Doch trotz eines besseren Verständnisses der Allergieentstehung gebe es noch keine umsetzbaren Strategien zur Primärprävention. Daher gelte es, in der sekundären und tertiären Prävention den Etagenwechsel von allergischer Rhinokonjunktivitis zum Asthma sowie das Fortschreiten von leichtem zu schwerem Asthma zu verhindern. Inhalative Kortikosteroide böten hier eine wirksame Therapieoption.

\section{Effektive Entzündungskontrolle} Asthma bronchiale tritt in Form vieler unterschiedlicher Endotypen auf, wie z.B. dem allergischen Asthma, erinner- te Dr. Mark Voss-Dirks, Hannover. Patienten mit allergischem Asthma profitierten in Phasen stärkerer entzündlicher Aktivität von einer höheren Steroiddosis.

Seit Januar 2016 ist für Asthmapatienten ab 18 Jahren die erste extrafeine Hochdosis-Fixkombination (Foster ${ }^{\circ}$ 200/6) aus dem inhalativen Kortikosteroid (ICS) Beclometason und dem langwirksamen $\beta_{2}$-Agonisten (LABA) Formoterol verfügbar. „Das Besondere an der neuen Hochdosistherapie ist, dass gezielt die Steroiddosis verdoppelt wurde, der LABA-Anteil hingegen unverändert bleibt", sagte Voss-Dirks. Dies biete einen Mehrwert für Patienten, die sensibel auf eine hohe LABA-Dosis reagieren, oder für Patienten mit einer infektgetriggerten Verstärkung der Entzündung in den Atemwegen.

Er schilderte den Fall einer Patientin, bei der sich unter einer Hochdosis-Fixkombination ICS/LABA zwar die Asth-

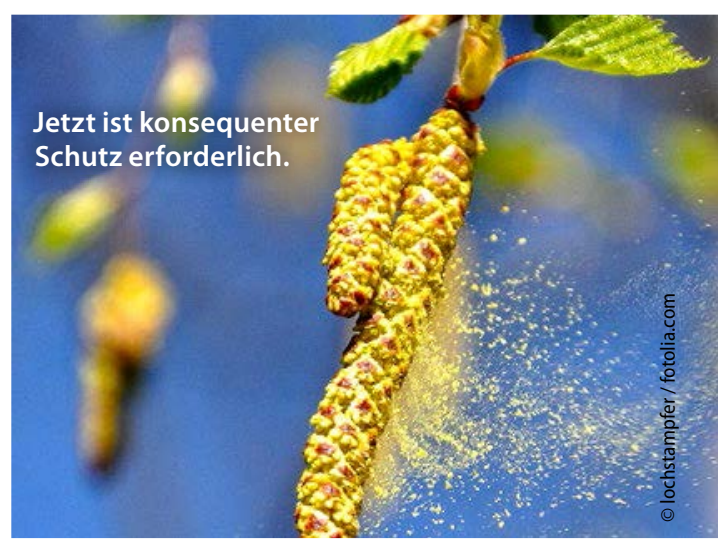

masymptome besserten, jedoch LABAvermittelte Nebenwirkungen wie Palpitationen, Schlaflosigkeit und Tremor auftraten. Nach Umstellung auf die neue Hochdosis mit doppelter Steroid- und gleichbleibender LABA-Komponente konnten eine effektive Entzündungskontrolle erreicht und die LABA-vermittelten Nebenwirkungen minimiert werden.

Susanne Pickl

- Pressetermin „Allergieprävalenz im Wandel - Herausforderung für die Asthmatherapie?", Kongress der Deutschen Gesellschaft für Pneumologie (DGP); Leipzig, März 2016 (Veranstalter: Chiesi) 